\title{
Human Autoantibodies against Desmoplakins in Paraneoplastic Pemphigus
}

\author{
Judith R. Oursler, Ramsey S. Labib, Lina Ariss-Abdo, Thomas Burke, Edward J. O'Keefe, ${ }^{\ddagger}$ and Grant J. Anhalt \\ Department of Dermatology, Johns Hopkins University, Baltimore, Maryland 21205; and ${ }^{\ddagger}$ Department of Dermatology, \\ University of North Carolina, Chapel Hill, North Carolina 27514
}

\begin{abstract}
Recently, a previously unrecognized autoantibody mediated blistering disease, paraneoplastic pemphigus has been described. Paraneoplastic pemphigus is associated with lymphoid malignancies, thymomas, and poorly differentiated sarcomas. Serum of affected patients contain pathogenic autoantibodies that immunoprecipitate from normal keratinocytes a characteristic complex of four polypeptides with $M_{\mathrm{r}}$ of $\mathbf{2 5 0}, \mathbf{2 3 0}, \mathbf{2 1 0}$, and $190 \mathrm{kD}$. As our preliminary studies indicated that the 250$\mathrm{kD}$ and the 210-kD antigens comigrated with desmoplakins I and II, we investigated the possibility that autoantibodies against the desmoplakins were a component of this autoimmune syndrome. 11 sera from affected patients were tested by indirect immunofluorescence against desmosome containing tissues, immunoprecipitation of metabolically labeled keratinocytes, and Western immunoblotting of desmoplakins I and II that had been purified to homogeneity from pig tongue epithelium. By indirect immunofluorescence, 9 of 11 sera showed strong binding to epithelial and nonepithelial desmosomes, and 2 were weakly reactive. All 11 immunoprecipitated 250- and 210-kD bands of variable intensity that comigrated with bands identified by a murine monoclonal antidesmoplakin antibody, and immunoblotting confirmed binding of the serum autoantibodies to purified desmoplakins. This demonstrates that paraneoplastic pemphigus is the first human autoimmune syndrome in which autoantibodies against the desmoplakins are a prominent component of the humoral autoimmune response. (J. Clin. Invest. 1992. 89:1775-1782.) Key words: pemphigus • cell adhesion - desmosomes - autoimmunity $\bullet$ paraneoplastic syndromes
\end{abstract}

\section{Introduction}

Human autoantibodies in bullous skin diseases such as pemphigus and pemphigoid have been instrumental in the identifcation of important structural proteins of the skin and mucosa. The use of these human reagents has provided much information about cell adhesion molecules of stratified squamous epithelia, desmosomal, and hemidesmosomal proteins (reviewed in reference 1).

A preliminary report of this work has appeared in abstract form (1991. Clin. Res. 39:195A).

Address reprint requests to Grant J. Anhalt, M. D., Department of Dermatology, Room 771 Ross Research Building, Johns Hopkins University, School of Medicine, 720 Rutland Avenue, Baltimore, MD 21205.

Received for publication 25 November 1991 and in revised form 23 January 1992.

J. Clin. Invest.

(c) The American Society for Clinical Investigation, Inc.

0021-9738/92/06/1775/08 $\$ 2.00$

Volume 89, June 1992, 1775-1782
Paraneoplastic pemphigus (PNP) ${ }^{1}$ is an autoimmune syndrome defined by the following criteria: (a) The presence of mucosal erosions and a polymorphous skin eruption, with papular lesions eventually progressing to blistering and erosive lesions affecting the trunk, extremities, and palms and soles in the context of an occult or known neoplasm. (b) Cutaneous histologic changes consisting of epidermal vacuolar interface dermatitis, keratinocyte necrosis, and intraepidermal cell-cell detachment (acantholysis). (c) The demonstration of IgG and complement components on the affected epithelial cell surfaces, and often granular/linear complement deposition along the basement membrane zone. $(d)$ Serum autoantibodies that bind to the cell surface of stratified squamous epithelia in a pattern common to all forms of pemphigus, but unlike other forms of pemphigus, these autoantibodies also bind to simple, columnar, and transitional epithelia. (e) These serum autoantibodies immunoprecipitate a complex of four high molecular weight proteins from keratinocytes, with estimated mol wt of $250,000,230,000,210,000$, and 190,000 (2). To date the neoplasms most commonly associated with paraneoplastic pemphigus are (in decreasing order of frequency) non-Hodgkin's lymphomas, chronic lymphocytic leukemia, thymomas, and poorly differentiated spindle cell sarcomas. The autoantibodies from these patients are pathogenic by passive transfer into neonatal mice, where they induce acantholysis of skin and esophageal mucosa.

The desmoplakins (desmoplakin I and II) are two major proteins located in the innermost portion of the desmosomal plaque. Their sequence and structure are known $(3,4)$ and desmoplakin II is apparently a product of alternate splicing of the desmoplakin I transcript (5). Desmoplakin I has a $M_{\mathrm{r}}$ $=250,000$ in SDS-PAGE and a calculated mol wt of 201,000. Desmoplakin II has a $M_{\mathrm{r}}=210,000$ in SDS-PAGE. Until now, no human autoantibodies against the desmoplakins had been found. Our study illustrates that human autoantibodies against the desmoplakins do indeed exist in paraneoplastic pemphigus and are a specific marker for the syndrome; however, the role of the antidesmoplakin antibodies in the pathogenesis of this autoimmune syndrome remains speculative at present.

\section{Methods}

The diagnostic criteria for classification as PNP in this study included the following criteria: an ulcerative and blistering mucocutaneous disease, associated with an underlying neoplasm, and autoantibodies against the characteristic antigen complex previously described (2). Serum was obtained from 11 patients with paraneoplastic pemphigus as so defined. Of these patients, the associated neoplasms were as follows: non-Hodgkin's lymphoma, $n=5$; chronic lymphocytic leukemia, $n=2$; poorly differentiated spindle cell sarcomas, $n=2$; benign thymoma, $n=1$, and Castleman tumor (giant lymph node hyperplasia,

1. Abbreviations used in this paper: DP, desmoplakin; PV, pemphigus vulgaris; PNP, paraneoplastic pemphigus. 
6), $n=1$. Additional antibodies used in the study included a murine monoclonal antibody specific for desmoplakins I and II (clone 2.15, Boehringer-Mannheim Corp., Indianapolis, IN), and as secondary antibodies where appropriate, a polyclonal rabbit anti-mouse IgG (MilesYeda Ltd., Israel), an affinity-purified FITC goat anti-human IgG, FITC goat anti-mouse IgG (Cappel Laboratories, West Chester, PA), and phycoerythrin anti-mouse IgG (Phycoprobe; Biomeda, Foster City, CA). Both the FITC anti-human IgG and the Phycoprobe were tested by Ouchterlony double immunodiffusion against human and mouse serum and by indirect immunofluorescence and were found to lack any interspecies cross-reactivity.

Indirect immunofluorescence was performed on tissues derived from BALB/c mice after cervical dislocation. The following tissues were embedded in optimal cutting temperature medium (Tissue-Tek; Miles Laboratories Inc., Elkhart, IN): esophagus, trachea, myocardium, small and large bowel, gall bladder, urinary bladder, kidney, and liver. Sections were first incubated with either dilutions of serum from patients, control subjects, or the murine monoclonal antibody to desmoplakin I and II followed by the addition of the appropriate secondary FITC anti-IgG. Slides were examined with a fluorescence microscope (Olympus, Tokyo, Japan). Control sera for immunofluorescence studies were obtained from patients with bullous pemphigoid, pemphigus vulgaris, pemphigus foliaceus, and normal human serum.

Indirect immunofluorescence was also performed on keratinocyte cultures using confocal microscopy. Keratinocytes were grown in chamber slides (Lab-Tek; Nunc, Naperville, IL) in keratinocyte growth medium (Clonetics Corp., San Diego, CA) to near confluence. They were then incubated in Dulbecco's MEM with $10 \%$ FCS (both from Sigma Chemical Co., St. Louis, MO) for $1 \mathrm{~h}$ at $37^{\circ} \mathrm{C}$, followed by sequential incubation with a dilution of serum from patient 6 and the monoclonal antidesmoplakin in the presence of $0.01 \%$ sodium azide (Sigma) at $4^{\circ} \mathrm{C}$ for $1 \mathrm{~h}$, followed by FITC anti-human IgG and phycoerythrin anti-mouse IgG, also at $4^{\circ} \mathrm{C}$.

Immunoprecipitation was performed according to the technique of Stanley et al. $(7,8)$. Human keratinocytes grown in keratinocyte growth medium to near confluence were incubated with ${ }^{14} \mathrm{C}$-labeled amino acids (New England Nuclear, Boston, MA) for $18 \mathrm{~h}$. Cultures were extracted in 0.5\% NP-40 (Calbiochem Corp., La Jolla, CA) in Tris-buffered saline with $2 \mathrm{mM}$ PMSF (Sigma), centrifuged at 100,000 $g$ for $1 \mathrm{~h}$, and the supernatant was dialyzed against $0.3 \%$ NP-40 in Tris-buffered saline. Labeled extracts were sequentially incubated with normal human serum, protein A-bearing staphylococci (Pansorbin; Calbiochem), test sera (from paraneoplastic patients, controls, or antidesmoplakin antibodies), and then staphylococcal protein G-agarose (ImmuBind; Genex, Gaithersburg, MD). Immunoprecipitated proteins were separated by SDS-PAGE, using a $5 \%$ slab gel, and the separated proteins were visualized by autoradiography. Control sera for immunoprecipitation were obtained from 15 patients with pemphigus vulgaris, 10 patients with pemphigus foliaceus, 3 patients with coexistent pemphigus vulgaris and adenocarcinomas of the bowel, 2 patients with coexistent pemphigus foliaceus and neoplasia (squamous-cell carcinoma in 1 patient and benign thymoma in the other), 10 patients with cicatricial pemphigoid, 15 with bullous pemphigoid, 2 with epidermolysis bullosa acquisita, 6 with linear IgA dermatitis, 10 with sys- temic lupus erythematosus with cutaneous involvement, 61 patients with erythema multiforme, 1 with dermatomyositis and ovarian carcinoma, 2 with cutaneous T-cell lymphoma, 2 with chronic myelogenous leukemia and "a rash", and 6 healthy subjects.

Epidermal extracts used in Western immunoblots were prepared from the skin of 1-2-d old BALB/c mice. After euthanasia, the epidermis was separated from the dermis by 40-s exposure to $0.02 \mathrm{M}$ EDTA (Sigma) in PBS at $56^{\circ} \mathrm{C} .1 \mathrm{~g}$ of epidermis was minced and sequentially homogenized in $5 \mathrm{ml}$ each of $1 \% \mathrm{NP}-40,1 \mathrm{M} \mathrm{NaCl}$, and $1 \%$ SDS (Bio-Rad Laboratories, Richmond, CA) with 5\% beta-mercaptoethanol (Bio-Rad) using a motor-driven Teflon-type Potter-Elvejem homogenizer. $2 \mathrm{mM}$ PMSF was added with each extraction, and the homogenate was centrifuged in a centrifuge (RC5B; Sorvall Instruments Div., DuPont Co., Newton, CT) at $4^{\circ} \mathrm{C}$ and $15,000 \mathrm{rpm}$ for $20 \mathrm{~min}$. Following the last extraction, $1 \mathrm{mM}$ PMSF, $10 \mathrm{mM}$ EDTA, and $2 \mathrm{mM}$ microbial protease inhibitors (Bio-Rad; pepstatin/chymostatin in DMSO, and antipain/leupeptin in water, 9) were added. The homogenate was then boiled for $3 \mathrm{~min}$, centrifuged, and the supernatant was aliquoted and frozen at $-70^{\circ} \mathrm{C}$.

Purification of the desmoplakins used in Western blots was performed according to the methods of O'Keefe (10). Briefly, desmosomes were isolated by extraction of pig tongue epithelium in sodium citrate buffer with $0.05 \%$ NP-40 in the presence of proteinase inhibitors, deoxyribonuclease, and ribonuclease. Desmoplakin isolation included extraction in $4 \mathrm{M}$ urea, followed by alternating ion exchange chromatography, gel filtration, and a second round of ion exchange chromatography. Analysis for purity to homogeneity was established by comigration with desmoplakins extracted from desmosomes, isoelectric point comparisons, peptide mapping and amino acid composition analysis, and reaction of the purified polypeptides with monoclonal antibody to desmoplakins.

All reagents used in Western blots were obtained from Sigma unless otherwise specified. All electrophoresis reagents were obtained from Bio-Rad Laboratories. SDS-PAGE in a borate-sulfate discontinuous system was performed as described by Neville (11). The total acrylamide concentration in the separation (lower) gel was $5 \%$, and the ratio of acrylamide to $N, N^{\prime}$-methylenebisacrylamide was 40:1. High molecular weight standards $(50,000-210,000$; Bio-Rad) were used for reference.

After separation by electrophoresis, proteins were transferred to nitrocellulose (Millipore Corp., Bedford, MA) as described by Towbin et al. (12). The nitrocellulose strips were incubated for $1 \mathrm{~h}$ with blocking solution (PTX; $150 \mathrm{mM} \mathrm{NaCl}, 10 \mathrm{mM} \mathrm{NaPO}_{4} \mathrm{pH}=7.5,1 \mathrm{mM}$ EGTA, $0.2 \%$ Triton $X, 0.006 \%$ NaAzide, $4 \%$ BSA), washed, and then probed with either patient sera, control sera, or murine monoclonal antibody to desmoplakins I and II. After further washes and blocking, the strips were incubated with ${ }^{125}$ I-staphylococcal protein A (NEN, Boston, MA), and visualized by autoradiography.

\section{Results}

Sera from patients with pemphigus, paraneoplastic pemphigus, and the monoclonal antidesmoplakin antibody all bound to

Figure 1. Fig. 1 demonstrates representative indirect immunofluorescence of four substrates, each substrate oriented horizontally by row: monkey esophagus $(A, B, C)$, murine small intestine $(D, E, F)$, murine myocardium $(G, H, I)$, and murine liver $(J, K, L)$. Primary antibodies included serum from a patient with pemphigus vulgaris (PV) as a "negative" control (vertically oriented by column, $A, D, G, J$ ), serum from a patient with paraneoplastic pemphigus $(P N P)-(B, E, H, K)$, and the murine monoclonal antidesmoplakin (anti-DP) as a positive control $(C, F, I, L)$. Secondary antibodies were FITC anti-human or FITC anti-murine IgG.

The cell surface of stratified squamous epithelium of monkey esophagus stained with PV sera $(A)$, PNP sera $(B)$, and anti-DP antibody $(C)$. PV autoantibodies failed to bind to all other tissues, including intestinal epithelium $(D)$, myocardium $(G)$, and liver $(J)$. However, both the PNP sera $(E)$, and anti-DP antibody $(F)$ show binding to the intestinal epithelium, with accentuation of binding in the subapical membrane where desmosomes are most concentrated. Both the PNP sera and anti-DP monoclonal bound the intercalated discs of myocardium $(H$ and $I$, respectively), and both also bound punctate structures on the hepatocyte cell surface and along bile canaliculi (shown as parallel punctate rows of dots, highlighted by the white arrows) that correspond precisely with the known distribution of desmosomes in liver. Bar $=20 \mu \mathrm{m}$. 

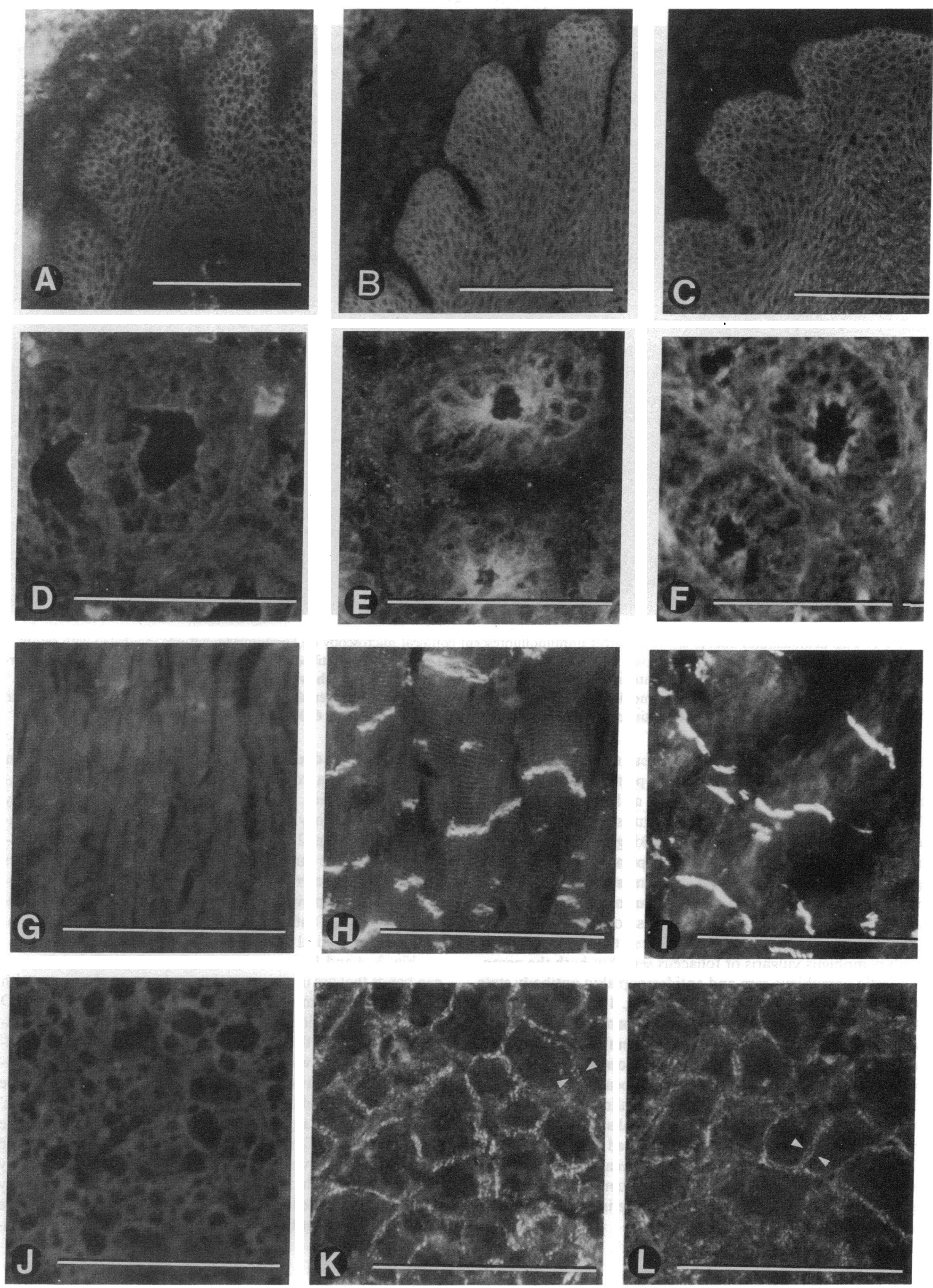


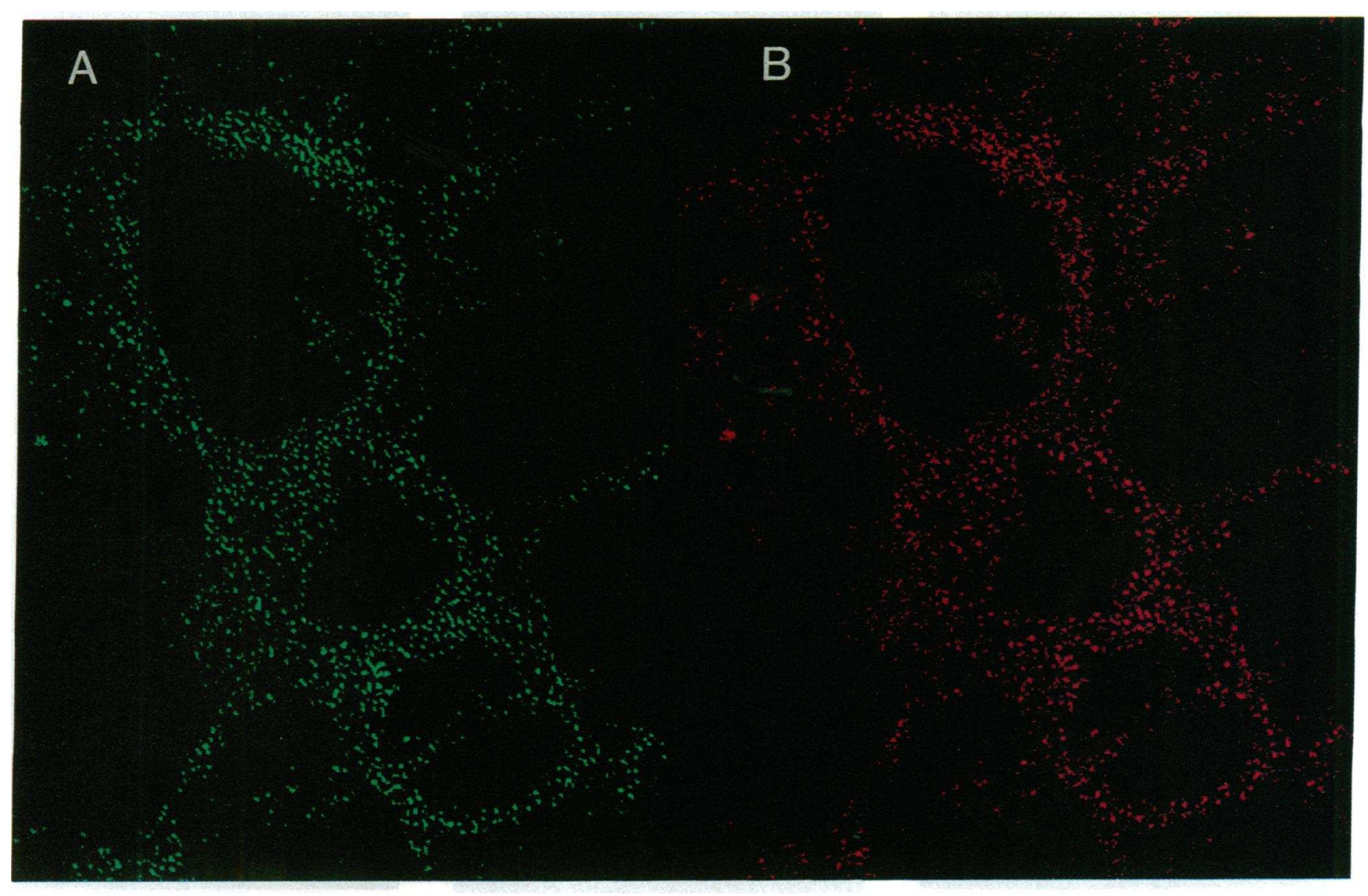

Figure 2. This demonstrates double-labeled indirect immunofluorescent confocal microscopy on keratinocyte cultures incubated with serum from patient $6(A)$ and the murine monoclonal anti-desmoplakin antibody $(B)$. Secondary antibodies were FITC anti-human IgG and phycoerythrin labeled anti-mouse IgG (both the anti-human and anti-mouse secondary antibodies showed no interspecies cross-reactivity). Both the human serum and the monoclonal antidesmoplakin bound identical discrete dots at the cell periphery of the keratinocytes, corresponding to the known distribution of desmosomes in keratinocyte cultures. ( $x-y$ axis, original magnification, 630 ; optical section $0.75-\mu \mathrm{m}$ thickness).

the cell surface of the stratified squamous epithelium of monkey esophagus in a similar pattern. The paraneoplastic sera and antidesmoplakin monoclonal did bind all layers of the epithelium more diffusely than the pemphigus sera, but this difference was quite subtle. In contrast, a striking difference was seen in the binding of autoantibodies from paraneoplastic pemphigus sera to simple epithelium of gastrointestinal mucosa (small intestine, colon, and gall bladder) and urinary bladder, as well as nonepithelial tissues in which desmosomes are present (myocardium, kidney, and liver). These tissues do not stain with pemphigus vulgaris or foliaceus sera, but both the paraneoplastic pemphigus sera and antidesmoplakin antibody stain these tissues in very similar patterns. Fig. 1 shows representative sections demonstrating binding of paraneoplastic sera to esophageal mucosa, to the subapical membrane of intestinal epithelia (where belt desmosomes are concentrated [13]), to the intercalated discs of myocardium, and focal structures on the hepatocyte cell surface and along bile canaliculi (corresponding to desmosomes stained by the monoclonal antibody). Of the 11 paraneoplastic pemphigus sera tested, 9 had unequivocal staining in the pattern described. Two had weaker reactivity with nonstratified squamous epithelia and could not be distinguished with complete certainty by these immunofluorescent criteria alone.
Fig. 2 shows double labeling on human keratinocytes in culture, using sera from a patient with paraneoplastic pemphigus and the murine monoclonal antidesmoplakin, viewed by confocal microscopy. Fig. $2 A$ is labeled with an FITC anti-human IgG and Fig. $2 B$ is labeled with a phycoerythrin antimurine IgG. One can see identical binding of both antibodies to discrete dots, corresponding to the distribution of the desmosomes at the periphery of the cultured cells. This distribution of binding of antidesmoplakin antibodies to cultured keratinocytes is identical to that reported by Ma et al. (14).

Fig. 3, $A$ and $B$, shows the complex and characteristic antigen bands that are immunoprecipitated by sera of patients with paraneoplastic pemphigus. With the exception of the $230-\mathrm{kD}$ bullous pemphigoid antigen, none of these bands were immunoprecipitated by the control sera from other diseases, including those patients with typical pemphigus vulgaris or pemphigus foliaceus. Heavily labeled 250 - and $210-\mathrm{kD}$ bands were precipitated by 9 of the 11 paraneoplastic pemphigus patients' sera, but not with any of the control sera. With two sera, these bands were only faintly visualized, and these were the same sera that reacted weakly with nonepithelial desmosomes by indirect immunofluorescence. The antidesmoplakin antibody immunoprecipitated bands that comigrated precisely with the $250-\mathrm{kD}$ band identified by these sera. The $210-\mathrm{kD}$ desmopla- 


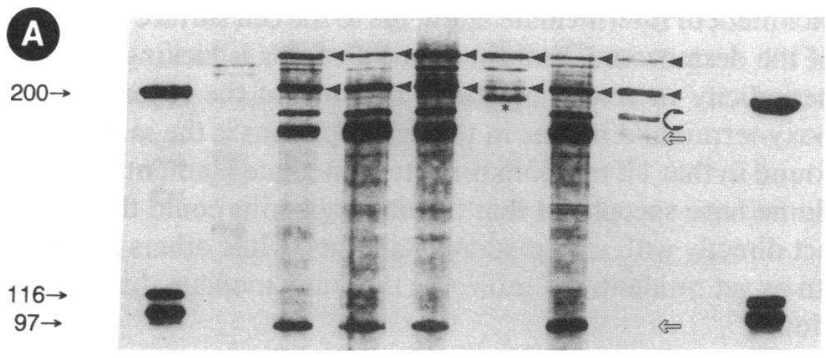

$\begin{array}{llllllll}\mathrm{BP} & 7 & 8 & 1 & \alpha \mathrm{DP} & 10 & 9 & \mathrm{PV}\end{array}$
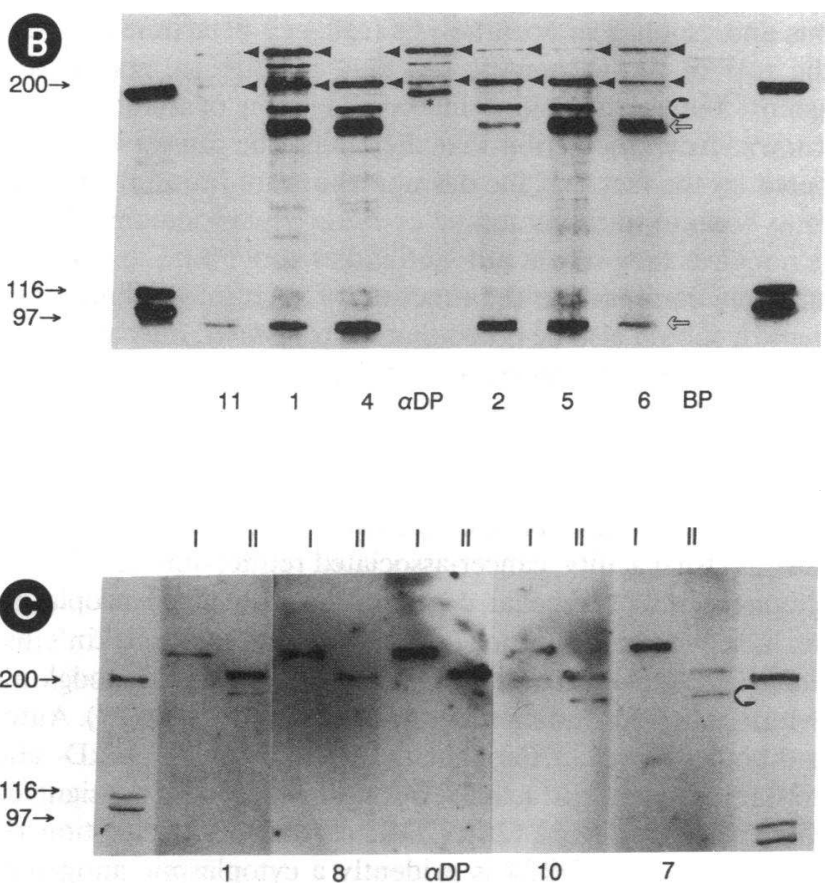

Figure 3. Figure 3, $A$ and $B$, demonstrates immunoprecipitation of metabolically labeled keratinocyte cultures by the sera of individual patients with paraneoplastic pemphigus. The numbers at the bottom of each lane correspond to the assigned patient case number. The controls include immunoprecipitates from the same extract produced by incubation with sera from patients with bullous pemphigoid $(B P)$, from pemphigus vulgaris $(P V)$, and a murine monoclonal antidesmoplakin antibody $(\alpha D P)$. Desmoplakin I and II bands are both marked by solid arrowheads. A band that comigrates precisely with desmoplakin I is clearly visualized by the monoclonal antibody and with variable intensity by sera from all patients with paraneoplastic pemphigus. Desmoplakin II is seen as a faint band that migrates just above the $200-\mathrm{kD}$ myosin marker, consistent with its migration in SDS-PAGE at $\sim 210 \mathrm{kD}$. This band is more heavily labeled by the patients' sera than the monoclonal for reasons that are not clear. The band recognized by the antidesmoplakin that migrates immediately below the myosin marker (asterisk) is only very faintly recognized by the patients' sera, and its identity is not clear. The band labeled by the shadowed arrowhead appears to be a degradation product of the purified desmoplakin II, as it is variably detected both by patients' sera and in the purified protein solution of DP II in immunoblotting. Finally, the identity of the lower molecular weight antigen bands (identified by the open arrows) that do not react with the antidesmoplakin antibody is currently unknown. $C$ is a Western immunoblot of purified desmoplakin I and II ( $I$ and $I I$ at the top of each lane) probed with PNP patients' sera (labeled by the case number) and the monoclonal antibody against desmoplakin I and II $(\alpha D P)$. All sera kin II is recognized as a faint discrete band with the monoclonal antibody, and as a much more heavily labeled band by the paraneoplastic patients' sera. The monoclonal anti-DP I and II monoclonal also recognizes a heavily labeled band that migrates just below the myosin molecular weight marker. It is not clear what this band represents, for it does not bind the monoclonal in the Western immunoblots (Fig. $3 C$ ) and it is also not precipitated by the patients' sera. There is also an apparent degredation product of the desmoplakin II polypeptide that is detected by both the patients' sera and in the Western immunoblots that migrates immediately below this band. The lower molecular weight bands that are apparent in the immunoprecipitates at $170 \mathrm{kD}$ and $90 \mathrm{kD}$ remain unidentified at present but are obvious in most samples. These bands were not visualized as clearly in the original description of this syndrome, and their importance is currently being investigated. The $230-\mathrm{kD}$ bullous pemphigoid antigen is detected by the control bullous pemphigoid serum used, and comigrating bands are obviously precipitated by only four of the eleven paraneoplastic pemphigus patients' sera.

The $130-\mathrm{kD}$ pemphigus vulgaris antigen is not visualized in these photographs, because of the extended autoradiographic exposure required to visualize this protein. The complex identified by paraneoplastic pemphigus sera is readily visualized after one week of exposure, but the pemphigus vulgaris antigen requires approximately three weeks of autoradiography for visualization. When this lengthy exposure was performed, the expected pemphigus antigen bands were seen with the control pemphigus sera; but no comigrating antigen bands were detected in the antigen complex identified by the paraneoplastic pemphigus sera (data not shown).

Western immunoblot analysis was performed with polypeptides extracted from neonatal murine epidermis probed with sera from 11 paraneoplastic pemphigus patients, and controls: one pemphigus vulgaris patient, two pemphigus foliaceus patients, one bullous pemphigoid patient, and three normal subjects. The antidesmoplakin antibody identified bands that comigrated precisely with the antigens at 250 and $210 \mathrm{kD}$. Fig. 3 $C$ shows Western immunoblotting with purified desmoplakin I and desmoplakin II in adjacent lanes, employing the patients' sera that had the most consistent reactivity in immunoblotting with whole murine epidermal extracts. Both purified desmoplakin I and desmoplakin II was bound by autoantibodies present in a total of 7 of the 11 PNP patients' sera. The patients' sera recognized the desmoplakin II band that migrated just above the myosin molecular weight marker, and the presumed degradation product of DP II described previously. Binding to the immobilized desmoplakins was more variable than that

stain both desmoplakins I and II. Desmoplakin II migrates just above the myosin marker, corresponding with the fine band identified by the same antibody in immunoprecipitation (Fig. 3, $A$ and $B$, solid arrowhead). There is also an apparent degradation product of desmoplakin II that is identified both in the immunoblots and by precipitation (Fig. 3, $A$ and $B$, shadowed arrowhead). The heavily labeled band immediately below the $210-\mathrm{kD}$ desmoplakin II band seen in the immunoprecipitates (asterisk) is not recognized in Western immunoblots. These final data confirm that PNP autoantibodies are specifically directed against the desmoplakins and are not nonimmunologically coprecipitated. 
demonstrated with the nondenatured polypeptides by immunoprecipitation.

\section{Discussion}

In this paper we have established that patients with the newly described syndrome, paraneoplastic pemphigus, mount a complex immune response which includes autoantibodies directed specifically against desmoplakins I and II. This specific affinity is suggested by indirect immunofluorescence studies that demonstrate binding to epithelial and nonepithelial desmosomes, by immunoprecipitation of high molecular weight antigens that comigrate with the desmoplakins, and is confirmed by binding of the antibodies to purified desmoplakin I and II by Western immunoblotting. The reactivity of patients' autoantibodies by immunoblotting establishes another important point, that the desmoplakins are not simply coprecipitated, but are targets of the autoantibodies. This is worth stressing, for in both pemphigus vulgaris and pemphigus foliaceus, a desmosomal plaque protein, plakoglobin (15) is nonimmunologically coprecipitated in immunoprecipitation techniques due to the presence of both reducible and nonreducible cross-linking to the pemphigus vulgaris and foliaceus antigens (16). Both the indirect immunofluorescent binding and the immunoblotting studies presented herein establish that this is not the case in paraneoplastic pemphigus, and the patients' autoantibodies are, in fact, directed against epitopes present on the desmosomal plaque proteins, desmoplakins I and II.

The reaction of patients' antibodies with the desmoplakins is not completely constant, and varies amongst individual patients' sera. As one might expect, we found that those patients' sera that react most strongly by immunofluorescence and immunoprecipitation also react most consistently by immunoblotting with purified desmoplakins. The most sensitive test for the presence of these autoantibodies appears to be immunoprecipitation of metabolically labeled keratinocytes. Unequivocal detection of the characteristic autoantibodies in paraneoplastic pemphigus by immunofluorescent criteria alone was possible in only 9 of the 11 patients in this study. As reported in our original description of the syndrome, in all cases we have tested to date, the autoantibodies were polyclonal and predominantly of the IgG 1 subclass (2).

The desmoplakins are major cytoskeletal structural proteins that have been localized to the innermost portion of the desmosomal plaque (reviewed in reference 17). Desmoplakin I has been found in all desmosomes studied $(13,18)$, whereas DP II has been found predominantly, but not exclusively, in stratified squamous epithelia (19). Overlapping cDNA clones encoding two major domains of the human desmoplakins have been identified (4), and suggest that DP I and II are encoded by separate mRNAs derived from a single gene (5). Analysis of these clones indicates that the central domain of DP I contains the heptad repeat characteristic of many $\alpha$ fibrous proteins and forms a coiled coil dimer of $\sim 130 \mathrm{~nm}$ in length. The carboxy terminus contains three regions each of which includes almost five repeats of a 38-residue motif. These regions are thought to assume globular conformations stabilized by intrachain ionic interactions, and have been found to have significant homology with regions of the $230-\mathrm{kD}$ bullous pemphigoid antigen.

It is thought that desmoplakins may play a role in the at- tachment of intermediate filaments to the cell surface at the site of the desmosome, but direct proof of this is lacking (3). The periodicity in acidic and basic residues of the repeating carboxy-terminal domains in the desmoplakins is the same as the found in that $1 \mathrm{~B}$ rod domain of intermediate filament proteins. Some have speculated that the desmoplakins could thus interact directly with intermediate filaments, while others postulate an as yet unidentified molecule that may mediate this interaction.

Although we have previously reported that passive transfer of whole immunoglobulin fractions from patients with paraneoplastic pemphigus into neonatal mice can induce cutaneous and esophageal acantholysis (cell-cell detachment, 1, 2), the role of the autoantibodies that are directed specifically against the desmoplakins in the induction of lesions is not known. Any speculation that they might be causative is tempered by the fact that the desmoplakins are intracellular proteins with no transmembrane or extracellular domains, and it is not clear how serum autoantibodies could bind to and presumably compromise the function of such intracellular antigens. It should also be noted that we transfused only autoantibodies, and any cell-mediated autoimmune tissue injury would not be reproduced in our passive transfer model.

Despite this, there are two striking "biological precedents" of paraneoplastic syndromes that are relevant to paraneoplastic pemphigus, paraneoplastic cerebellar degeneration (20), and loss of visual acuity, cancer-associated retinopathy $(21,22)$. In paraneoplastic cerebellar degeneration, associated neoplasms include ovarian carcinoma (most common, 23), Hodgkin's disease (24), ductal carcinoma of the breast (25), non-Hodgkin's lymphoma (26), and mesodermal ovarian sarcoma (27). Autoantibodies found in the patients' sera react with $34 \mathrm{kD}$ - and 62-kD antigens extractable from Purkinje cells (designated CDR34 and CDR62 [28]; CDR, cerebellar degeneration related-antigen). CDR34 is evidently a cytoplasmic antigen of the endoplasmic reticulum of neuroectodermal tissue (29) and it is anomalously expressed only by the ovarian carcinoma cells from patients with the paraneoplastic cerebellar degeneration and is not present in similar tumors from unaffected subjects (30). Cancer-associated retinopathy is usually associated with small cell carcinoma of the lung (21) and autoantibodies that bind antigens found within the nuclei of cells of the retinal pigment epithelium, the choroid, and choroidal vessels (31, 32). Passive transfer of human autoantibodies into rodents produces vesicular degradation of optic nerve myelin sheaths (32) and reduction of retinal ganglion cells (33) in vivo. Unlike the cerebellar degeneration syndrome, however, these autoantigens can be extracted both from tumor of both affected and unaffected individuals and from the human small cell lung carcinoma cell line $119(32,34)$.

It is proposed that an "autoimmune" paraneoplastic syndrome results in part from the immunologic response of the host to the tumor. According to this hypothesis, the host produces antitumor antibodies that react with the tumor and may limit its growth. The antibodies may also gain access to and cross-react with antigens on the specific target tissue and compromise its function. For this argument to be persuasive, it must be shown that the patient produces antibodies that react with tumor cells, that these antibodies bind in vivo to components of normal host tissue, and compromise the physiologic 
function of that organ. These three conditions have been met for paraneoplastic cerebellar dysfunction and cancer-associated retinopathy, but only two of these criteria have been established in the case of paraneoplastic pemphigus.

We do not know if autoantibodies in these patients' sera react with tumor antigens from affected individuals. This line of investigation has been hampered by the lack of tumor tissue available for study, as this is a newly recognized syndrome and most cases were recognized retrospectively after the death of the affected individual. It is known that desmoplakins are expressed in thymomas and Castleman tumors, and it is likely that the patients' autoantibodies will react with these tumors. What is curious is that the majority of affected patients have lymphomas or chronic leukemias of B cell origin, and it is generally accepted that these cells do not produce desmosomes or express desmoplakins.

However, the hypothesis that there may be a link between the immune response against lymphoid neoplasms and desmosomal proteins finds some basis in a review of the literature. It has been reported that, paradoxically, primitive tumors may produce junctions that are not normally present in the tissue from which the tumor originated. Desmosomes and "desmosome-like" cell junctions have been known to be anomalously produced by tumors that do not normally posses desmosomes. The list of tumors that are known to possess these desmosomelike structures (by morphologic criteria) includes sarcomas, lymphomas, neuroblastomas, and meningiomas $(35,36,37)$. In addition, the desmoplakins have been detected by immunostaining in reactive lymph nodes, tonsils, non-Hodgkin's B cell lymphomas, and synovial sarcomas (38). These data provide some basis for our speculation that these tumors may anomalously produce antigens that are cross-reactive with epithelial antigens, and the autoantibodies directed against tumor antigen cause the mucocutaneous disease, but direct proof is lacking. Arguing against this hypothesis is the notable absence of paraneoplastic pemphigus in patients affected with much more common tumors that also express desmoplakins and other epithelial antigens such as squamous cell and basal cell carcinomas. Therefore, a more complex and as yet unexplored interaction between these lymphoid tumors and the host may be necessary to induce this rare phenomenon.

In conclusion, patients with paraneoplastic pemphigus produce antibodies, specifically desmoplakins I and II. The role of these antibodies in the pathogenesis of tissue damage in the syndrome is not yet known, and their role as a component of the antitumor immune response remains to be investigated.

\section{Acknowledgments}

The authors would like to thank Dr. J. Clark Huff for kindly providing sera from patients with erythema multiforme, and Ms. Paula Bonitz for her skilled technical assistance.

This work was supported in part by the Stetler Research Fund for Women Physicians (J. R. Oursler), and National Institutes of Health grants RO1-AR-32490, RO1-AR-40018, and KO4-AR01686 (G. J. Anhalt) and RO1-AR-25871 (E. J. O'Keefe), and by an educational grant from the Sandoz Pharmaceutical Corp.

\section{References}

1. Stanley, J. R. 1989. Pemphigus and pemphigoid as paradigms of organ-specific autoantibody-mediated diseases. J. Clin. Invest. 83:1443-1448.

2. Anhalt, G. J., S. Kim, and J. R. Stanley. 1990. Paraneoplastic pemphigus: an autoimmune mucocutaneous disease associated with neoplasia. $N$. Engl. $J$. Med. 323:1729-1735.

3. O'Keefe, E. J. H. P. Erickson, and V. Bennet. 1989. Desmoplakin I and desmoplakin II purification and characterization. J. Biol. Chem. 264:8310-8318. 4. Green, K. J., D. A. Parry, P. M. Steiner, M. L. A. Virata, R. M. Wagner, B. D. Angst, and L. A. Nilles. 1990. Structure of the human desmoplakin implications for function in the desmosomal plaque. J. Biol. Chem. 265:2603-2612.

5. Green, K. J., R. D. Goldman, and R. L. Chisholm. 1988. Isolation of cDNAs encoding desmosomal plaque proteins: evidence that bovine desmoplakins I and II are derived from two mRNAs and a single gene. Proc. Natl. Acad. Sci. USA. 85:2613-2617.

6. Castleman, B., and V. W. Towe. 1954. Case records of the Massachusetts General Hospital. Weekly clinicopathological exercise, case 40011. N. Engl. J. Med. 250:26-30.

7. Stanley, J. R., L. Koulu, and C. Thivolet. 1984. Distinction between epidermal antigens binding pemphigus vulgaris and pemphigus foliaceus autoantibodies. J. Clin. Invest. 74:313-320.

8. Stanley, J. R., M. Yaar, P. Hawley-Nelson, and S. I. Katz. 1982. Pemphigus antibodies identify a cell surface glycoprotein synthesized by human and mouse keratinocytes. J. Clin. Invest. 70:281-288.

9. Labib, R. S., G. J. Anhalt, H. P. Patel, D. F. Mutasim, and L. A. Diaz. 1986. Molecular heterogeneity of the bullous pemphigoid antigens as detected by immunoblotting. J. Immunol. 136:1231-1235.

10. O'Keefe, E. J., H. P. Erickson, and V. Bennett. 1989. Desmoplakin I and desmoplakin II purification and characterization. J. Biol. Chem. 264:8310-8318.

11. Nelville, D. M. 1971. Molecular-weight determination of protein-dodecylsulfate complexes by gel electrophoresis in a discontinuous buffer system. J. Biol. Chem. 246:6328-6334.

12. Towbin, H., T. Staehelin, and J. Gordon. 1979. Electrophoretic transfer of proteins from polyacrylamide gels to nitrocellulose sheets: procedure and some applications. Proc. Natl. Acad. Sci. USA. 76:4350-4354.

13. Franke, W. W., E. Schmid, C. Grund, H. Muller, I. Englebrecht, R. Moll, J. Dtasler, and E.-D. Jarasch. 1981. Antibodies to high molecular weight polypeptides of desmosomes: specific localization of a class of junctional proteins in cells and tissues. Differentiation. 20:217-241.

14. Ma, A. S. P., and A. L. Lorincz. 1988. Immunofluorescence localization of peripheral proteins in cultured keratinocytes. J. Invest. Dermatol. 90:331-335.

15. Cowin, P., H.-P. Kapprell, W. W. Franke, J. Tamkun, and R. Hynes. 1986. Plakoglobin: a protein common to different kinds of intercellular adhering junctions. Cell. 46:1063-1073.

16. Korman, N. J., R. W. Eyre, V. Klaus-Kovtun, and J. R. Stanley. 1989. Demonstration of an adhering-junction molecule (plakoglobin) in the autoantigens of pemphigus foliaceus and pemphigus vulgaris. N. Engl. J. Med. 321:631635.

17. Schwartz, M. A., K. Oaribe, J. Kartenbach, and W. W. Franke. 1990. Desmosomes and hemidesmosomes: constitutive molecular components. Annu. Rev. Cell Biol. 6:461-491.

18. Franke, W. W., R. Moll, D. L. Schiller, E. Schmid, J. Kartenbeck, and H. Meuller. 1983. Desmoplakins of epithelial and myocardial desmosomes are immunologically and biochemically related. Differentiation. 23:115-127.

19. Angst, B. D., L. A. Nilles, and K. J. Green. 1990. Desmoplakin II expression is not restricted to stratified epithelia. J. Cell Sci. 97:247-257.

20. Greenlee, J. E., and H. L. Lipton. 1986. Anticerebellar antibodies in serum and cerebrospinal fluid of a patient with oat cell carcinoma of the lung and paraneoplastic cerebellar degeneration. Ann. Neurol. 19:82-85.

21. Kornguth, S., R. Klein, R. Appen, and J. Choate. 1982. Occurrence of anti-retinal ganglion cell antibodies in patients with small cell carcinoma of the lung. Cancer (Phila.). 50:1289-1293.

22. Grunwald, G. B., R. Klein, M. A. Simmonds, and S. E. Kornguth. 1985. Autoimmune basis for visual paraneoplastic syndrome in patients with small-cell carcinoma. Lancet. i:658-661.

23. Brashear, H. R., J. E. Greenlee, K. A. Jaeckle, and J. W. Rose. 1989. Anticerebellar antibodies in neurologically normal patients with ovarian neoplasms. Neurology. 39:1605-1609.

24. Schlake, H. P., I. W. Husstedt, K. H. Grotemeyer, and P. R. Otter. 1989. Paraneoplastic subacute cerebellar degeneration in Hodgkin's disease. Report of three cases and review of the literature. Clin. Neurol. Neurosurg. 91:329-335.

25. Tsukamoto, T., H. Yamamoto, Y. Iwasaki, O. Yoshie, H. Terunuma, and H. Suzuki. 1989. Antineural autoantibodies in patients with paraneoplastic cerebellar degeneration. Arch. Neurol. 46:1225-1229.

26. Smith, J. L., J. C. Finley, and V. A. Lennon. 1988. Autoantibodies in paraneoplastic cerebellar degeneration bind to cytoplasmic antigens of Purkinje cells in humans, rats and mice and are of multiple immunoglobulin classes. $J$. Neuroimmunol. 18:37-48.

27. Greenlee, J. E., H. R. Brashear, and R. M. Herndon. 1988. Immunoperox idase labelling of rat brain sections with sera from patients with paraneoplastic cerebellar degeneration and systemic neoplasia. J. Neuropathol. \& Exp. Neurol. 47:561-571. 
28. Anderson, N. E., M. K. Rosenblum, and J. B. Posner. 1988. Paraneoplastic cerebellar degeneration: clinical-immunological correlations. Ann. Neurol. 24:559-567.

29. Rodriguez, M., L. I. Truh, B. P. O'Neill, and V. A. Lennon. 1988. Autoimmune paraneoplastic cerebellar degeneration: ultrastructural localization of antibody-binding sites in Purkinje cells. Neurology. 38:1380-1386.

30. Furneaux, H. M., M. K. Rosenblum, J. Dalmau, E. Wong, P. Woodruff, F. Graus, and J. B. Posner. 1990. Selective expression of Purkinje-cell antigens in tumor tissue from patients with paraneoplastic cerebellar degeneration. $N$. Engl. J. Med. 322:1844-1851.

31. Thirkill, C. E. P. FitzGerald, R. C. Sergott, A. M. Roth, N. K. Tyler, and J. L. Keltner. 1989. Cancer-associated retinopathy (CAR syndrome) with antibodies reacting with retinal, optic-nerve, and cancer cells. N. Engl. J. Med. 321:15671571.

32. Kornguth, S. E., T. Kalinke, G. B. Grunwald, H. Schutta, and D. Dahl. 1986. Anti-neurofilament antibodies in the sera of patients with small cell carcinoma of the lung and with visual paraneoplastic syndrome. Cancer Res. 46:25882595.

33. Kornguth, S. E., P. D. Spear, and E. Langer. 1982. Reduction in numbers of large ganglion cells in cat retina following intravitreous injection of antibodies. Brain Res. 245:35-45.

34. Grunwald, G. B., S. E. Kornguth, J. Towfighi, J. Sassani, M. A. Simmonds, C. M. Housman, and N. Papadopoulos. 1987. Autoimmune basis for visual paraneoplastic syndrome in patients with small cell lung carcinoma. Retinal immune deposits and ablation of retinal ganglion cells. Cancer (Phila.). 60:780-786.

35. Erlandson, R. A. 1981. Diagnostic transmission electron microscopy of human tumors. Masson Publishing USA Inc., New York. 107-116.

36. Ghadially, F. N. 1982. Ultrastructural pathology of the cell matrix. Second ed. Butterworth \& Co., Ltd., London. 1-947.

37. Geiger, B., E. Schmid, and W. W. Franke. 1983. Spatial distribution of proteins specific for desmosomes and adherens junctions in epithelial cells demonstrated by double immunofluorescence microscopy. Differentiation. 23:189205.

38. Miettinen, M. 1991. Keratin subsets in spindle cell sarcomas. Keratins are widespread but synovial sarcoma contains a distinctive keratin polypeptide pattern and desmoplakin. Am. J. Pathol. 138:505-513. 